\title{
ABBREVIATIONS AND ACRONYMS
}

$\begin{array}{ll}\text { ANC } & \text { African National Congress } \\ \text { CCP } & \text { Common Commercial Policy } \\ \text { CDU } & \text { Christian Democratic Union (Germany) } \\ \text { CSU } & \text { Christian Social Union (Germany) } \\ \text { CESDP } & \text { Common European Security and Defence Policy } \\ \text { CFSP } & \text { Common Foreign and Security Policy } \\ \text { CSCE } & \text { Conference on Security and Cooperation in Europe } \\ \text { CJTF } & \text { Combined Joint Task Forces } \\ \text { CoCom } & \text { Coordination Committee on Multilateral Export Controls } \\ \text { COMECON } & \text { Council for Mutual Economic Assistance } \\ \text { CTBT } & \text { Comprehensive Test Ban Treaty } \\ \text { CTBTO } & \text { Comprehensive Nuclear Test Ban Treaty Organisation } \\ \text { DDR } & \text { Deutsche Demokratische Republik (East Germany) } \\ \text { EAPC } & \text { Euro-Atlantic Partnership Council } \\ \text { ECB } & \text { European Central Bank } \\ \text { ECJ } & \text { European Court of Justice } \\ \text { ECSC } & \text { European Coal and Steel Community } \\ \text { ECU } & \text { European Currency Unit } \\ \text { EDC } & \text { European Defence Community } \\ \text { EEA } & \text { European Economic Area } \\ \text { EEC } & \text { European Economic Community } \\ \text { EFTA } & \text { European Free Trade Association } \\ \text { EMU } & \text { Economic and Monetary Union } \\ \text { ENDC } & \text { Eighteen Nation Disarmament Commission (UN) } \\ \text { EP } & \text { European Parliament } \\ \text { EPC } & \text { European Political Cooperation } \\ \text { ERRF } & \text { European Rapid Reaction Force } \\ \text { ESDI } & \text { European Security and Defence Identity } \\ \text { ESDP } & \text { European Security and Defence Policy } \\ \text { ESF } & \text { European Social Fund }\end{array}$




$\begin{array}{ll}\text { ETUC } & \text { European Trade Union Confederation } \\ \text { FDP } & \text { Free Democratic Party (Germany) } \\ \text { FPÖ } & \text { Freuheitliche Partei Österreichs (Austrian Freedom Party) } \\ \text { GATT } & \text { General Agreement on Tariffs and Trade } \\ \text { GDP } & \text { Gross Domestic Product } \\ \text { GNI } & \text { Gross National Income } \\ \text { GNP } & \text { Gross National Product } \\ \text { IFOR } & \text { Implementation Forces } \\ \text { IGC } & \text { Intergovernmental Conference } \\ \text { IMF } & \text { International Monetary Fund } \\ \text { INF } & \text { Intermediate Range Nuclear Forces } \\ \text { IR } & \text { International Relations } \\ \text { IUI } & \text { Industriens Utredningsinstitut (Research Institute of Industrial } \\ & \text { Economics, Sweden) } \\ \text { KD } & \text { Kristdemokraterna (Christian Democrats, Sweden) } \\ \text { KFOR } & \text { Kosovo Implementation Force } \\ \text { LMB } & \text { Labour Market Board } \\ \text { LN } & \text { Lega Nord (Northern League, Italy) } \\ \text { LO } & \text { Landsorganisationen Sverige (Swedish Trade Union } \\ & \text { Confederation) } \\ \text { MEP } & \text { Member of the European Parliament } \\ \text { MP } & \text { Member of Parliament } \\ \text { MFA } & \text { Ministry of Foreign Affairs } \\ \text { MPLA } & \text { Movement for the Popular Liberation of Angola } \\ \text { NACC } & \text { North Atlantic Cooperation Council } \\ \text { NATO } & \text { North Atlantic Treaty Organisation } \\ \text { NIB } & \text { Nämnden för internationellt bistånd (Committee for } \\ & \text { International Assistance, Sweden) } \\ \text { NIEO } & \text { New International Economic Order } \\ \text { NGO } & \text { Non-Governmental Organisation } \\ \text { N+N } & \text { Neutral and Non-Aligned } \\ \text { NORDCAPS } & \text { Nordic Coordinated Arrangement for Military Peace Support } \\ \text { NORDEK } & \text { Nordic Economic Community } \\ \text { NPT } & \text { Non-Proliferation Treaty } \\ \text { NSC } & \text { National Security Council } \\ \text { NWFZ } & \text { Nuclear Weapon-Free Zone } \\ \text { ODA } & \text { Overseas development assistance } \\ \text { OECD } & \text { Organisation for Economic Cooperation and Development } \\ \text { OPEC } & \text { Organisation of Petroleum Exporting Countries } \\ \text { OSCE } & \text { Organisation for Security and Cooperation in Europe } \\ \text { ÖVP } & \text { Österreichs Volkspartei (Austrian People's Party) } \\ \text { PAIGC } & \text { African Party for the Independence of Guinea and Cape Verde }\end{array}$




\begin{tabular}{|c|c|}
\hline PANA & Peace and Neutrality Alliance (Ireland) \\
\hline PARP & Planning and Review Process \\
\hline PES & Party of European Socialists \\
\hline PFP & Partnership for Peace \\
\hline PLO & Palestinian Liberation Organisation \\
\hline PTBT & Partial Test Ban Treaty \\
\hline PTK & $\begin{array}{l}\text { Privattjänstmänna-kartellen (salaried employees in private } \\
\text { industry, Sweden) }\end{array}$ \\
\hline QMV & Qualified Majority Voting \\
\hline RRF & Rapid Reaction Force \\
\hline SACO & $\begin{array}{l}\text { Sveriges Akademikers Centralorganisation (Swedish } \\
\text { Confederation of Professional Associations) }\end{array}$ \\
\hline SAF & $\begin{array}{l}\text { Svenska Arbetsgivareföreningen (Swedish Employers' } \\
\text { Association) }\end{array}$ \\
\hline SALT & Strategic Arms Limitation Talks \\
\hline SAP & $\begin{array}{l}\text { Socialdemokratiska arbetarepartiet (The Social Democratic } \\
\text { Party of Sweden) }\end{array}$ \\
\hline SDU & Scandinavian Defence Union \\
\hline SEA & Single European Act \\
\hline SEK & Swedish Krona \\
\hline SFOR & Stabilisation Forces \\
\hline SI & Sveriges Industriförbundet (Swedish Association of Industries) \\
\hline SIDA & Swedish International Development Authority \\
\hline SPÖ & Sozialistische Partei Österreichs (Austrian Socialist Party) \\
\hline SNS & $\begin{array}{l}\text { Studieförbundet Näringsliv och Samhälle (Centre for Business } \\
\text { and Policy Studies, Sweden) }\end{array}$ \\
\hline SSBN & Ballistic Missile Nuclear Submarine \\
\hline START & Strategic Arms Reduction Treaty \\
\hline SWAPO & South West Africa People's Organisation \\
\hline TCO & $\begin{array}{l}\text { Tjänstemännens Centralorganisationen (Central Organisation } \\
\text { of Salaried Employees, Sweden) }\end{array}$ \\
\hline TFCMA & Treaty of Friendship, Cooperation and Mutual Assistance \\
\hline UNCED & United Nations Conference on Environment and Development \\
\hline UNEF & United Nations Emergency Force \\
\hline UNICEF & United Nations Children's Emergency Fund \\
\hline UNIDO & United Nations Industrial Development Organisation \\
\hline UNIFIL & United Nations Interim Force in Lebanon \\
\hline USSR & Union of Soviet Socialist Republics \\
\hline $\mathrm{VF}$ & $\begin{array}{l}\text { Verkstadsföreningen (Association of Engineering Industries, } \\
\text { Sweden) }\end{array}$ \\
\hline $\mathrm{W}$ & Western European Armaments Group \\
\hline & Western European Union \\
\hline
\end{tabular}

